\title{
Slow Ventricular Tachycardia Located in the Epicardium of the Left Ventricular Base and Characterized by Effects of Adenosine Triphosphate, Nicorandil and Verapamil
}

\author{
Youichi Kobayashi, MD; Akira Miyata, MD; Kaoru Tanno, MD; \\ Shuji Kikushima, MD; Takao Baba, MD; Takashi Katagiri, MD
}

\begin{abstract}
A 31-year-old male with slow ventricular tachycardia (VT) developed a nonsustained VT with prolongation of the JT intervals after injection of contrast medium and saline into the marginal vein of the coronary sinus. The earliest activation site of the VT existed in the epicardium of the left ventricular base. Adenosine triphosphate prevented induction of VT and prolongation of JT intervals. Ventricular premature contractions showing the same morphology as the VT were also inhibited by nicorandil and verapamil. The mechanism of the VT was suggested to be abnormal automaticity due to an increase in the $\mathrm{Ca}^{++}$current into cells after prolongation of the action potential duration induced by hypothermia. (Jpn Circ J 1998; 62: 947-951)
\end{abstract}

Key Words: Adenosine triphosphate; Epicardium; Nicorandil; Slow ventricular tachycardia; Verapamil

W present a case where slow ventricular tachycardia (VT) was presumably due to abnormal automaticity, the focus of which was located in the epicardium of the left ventricular (LV) base. The slow VT had specific pharmacological characteristics; that is, sensitivity to adenosine triphosphate (ATP), nicorandil and verapamil.

\section{Case Report}

A 31-year-old male had slow VT during an operation to repair a jaw fracture. He was referred to our clinic for closer examination. Physical examination and chest X-ray were both normal. The baseline of the ECG showed an incomplete right bundle branch block (RBBB). A Holter monitor showed 158 episodes of 3 or more consecutive ventricular premature contractions (VPCs) of which the longest VT lasted about $5 \mathrm{~min}$ with a slow rate of 75-95 beats/min (Fig 1). Exercise testing did not induce VT and no changes were shown in the ST and T waves.

Cardiac catheterization revealed normal coronary arteries and normal intracardiac pressures. Left ventriculography showed a normal ejection fraction (61.5\%), although the septum, anterolateral wall, and posterolateral wall were hypokinetic. Endomyocardial biopsy revealed mild hypertrophic myocardial cells with mild degeneration.

\section{Electrophysiological Study}

Informed written consent was obtained before the electrophysiological study. Clinical VT could not be induced by programmed atrial and ventricular stimulation even under isoproterenol provocation, but a nonclinical poly-

(Received June 2, 1998; revised manuscript received August 28, 1998; accepted September 3, 1998)

Third Department of Internal Medicine, Showa University School of Medicine, Tokyo, Japan

Mailing address: Youichi Kobayashi, MD, The Third Department of Internal Medicine, Showa University School of Medicine, 1-5-8 Hatanodai, Shinagawa-ku, Tokyo 142-8666, Japan morphic VT was induced. When contrast medium was injected into the marginal vein of the coronary sinus (CS), which was located at the LV base, slow VT showing a RBBB pattern was induced with warm-up phenomenon (Fig 2, left panel; Fig 3, upper panel). However, the injection of contrast medium into the apical marginal vein of the CS could not induce VT (Fig 2, right panel). The slow VT induction by contrast medium was reproducible, and VT was also induced by injection of physiological saline solution into the vein (Fig 3, middle panel). Electrogram recording from the distal tip of the CS catheter preceded the QRS by $-31 \mathrm{~ms}$ (Fig 3, lower panel). These findings strongly suggested that the focus of the VT was located in the epicardium of the posterior wall in the LV base. We then tried to evaluate the efficacy of ATP on the VT using injections of mixtures of ATP $(5 \mathrm{mg})$ with saline, and ATP with contrast medium (Fig 4). As shown in Fig 4, both mixtures prevented induction of the VT. Table 1 shows the JT intervals in lead II at VT induction and prevention. The mean values of 3-7 RR and JT intervals were calculated at $400 \mathrm{~mm} / \mathrm{s}$ of paper speed in the control state, just before VT induction, and around $10 \mathrm{~s}$ after ATP injection. JT intervals were prolonged just before VT induction by injection of saline and contrast medium. ATP prevented the prolongation of JT intervals.

\section{Drug Interventions}

On separate days we assessed the efficacy of a $\mathrm{K}^{+}$ channel opener, nicorandil, and a $\mathrm{Ca}^{++}$antagonist, verapamil. Electrocardiograms were recorded for more than 30 min and we confirmed frequent VPCs with the same morphology as the VT. Nicorandil $(12 \mathrm{mg})$ was infused intravenously over $3 \mathrm{~min}$ and the electrocardiograms were observed for $40 \mathrm{~min}$. As shown in lines 1 and 2 of Fig 5, 81 VPC were observed in $10 \mathrm{~min}$ in the control state. As shown in lines 5-8, after around $10 \mathrm{mg}$ of nicorandil, VPCs were being reduced and completely disappeared after 12 $\mathrm{mg}$. Then, $6 \mathrm{~min}$ later, the VPC reappeared in lines 9 and 10 , because the half life of nicorandil is approximately 8 


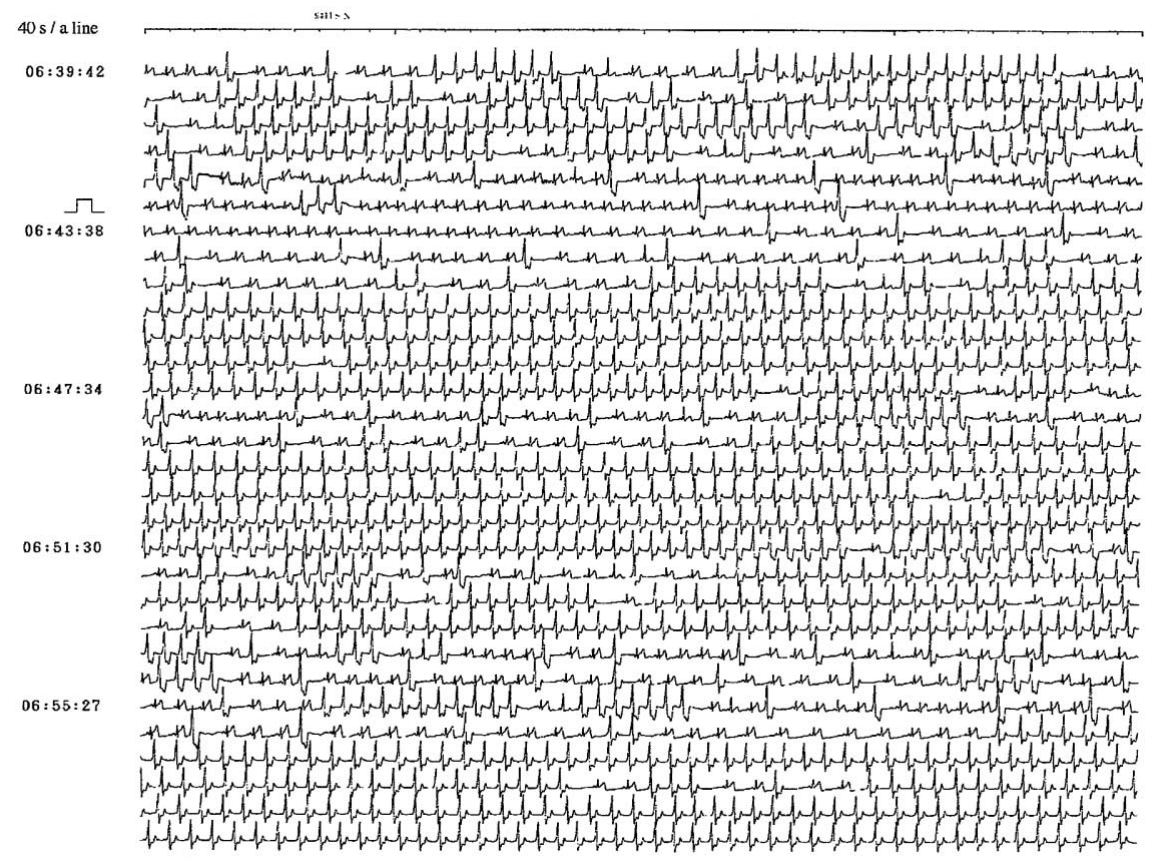

Fig 1. Holter monitor recording of slow ventricular tachycardia (VT) showing frequent VPCs and VT of which the VT lasted a few minutes with a slow rate of $75-95$ beats $/ \mathrm{min}$. Note that sinus arrhythmia was also seen, suggesting unbalanced autonomic nervous activity.
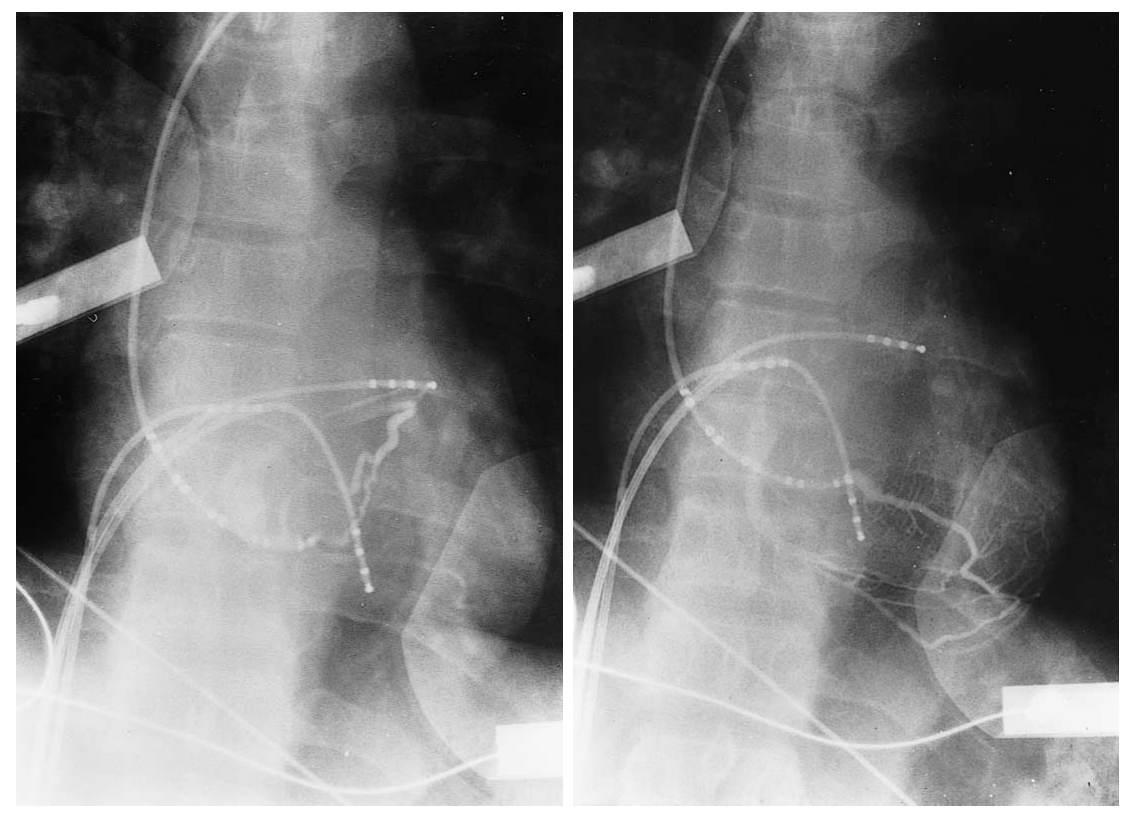

Fig 2. Angiography of the coronary sinus (CS). Slow ventricular tachycardia (VT) was induced when contrast medium was injected into the marginal vein of the CS, which was located at the left ventricular base (left panel). However, the injection of contrast medium into the apical marginal vein of the CS could not induce VT (right panel). min. Finally, nicorandil suppressed $65.6 \%$ of the VPCs in $10 \mathrm{~min}$. Verapamil $(10 \mathrm{mg})$ completely suppressed the VPCs (control 89 vs verapamil 0 in 30 min: reduction rate $100 \%)$. We considered that nicorandil and verapamil were effective in preventing the VPCs.

\section{Discussion}

The slow VT was characterized as follows: (1) it was induced, with prolongation of the JT intervals, by contrast medium and saline at room temperature; (2) it was inhibited, with prevention to the JT prolongation, by ATP; (3) an 

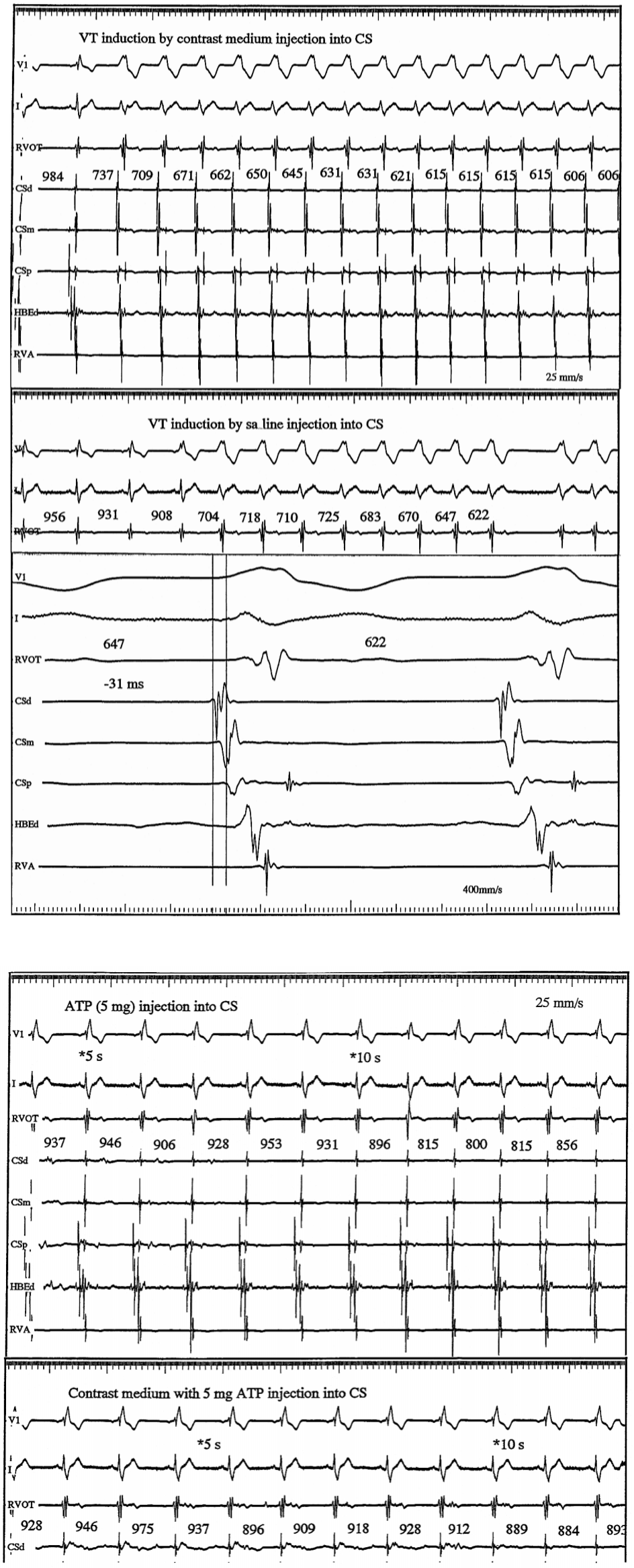

Fig 3. Ventricular tachycardia (VT) induction by injection of contrast medium and saline into the vein. VT showing a right bundle branch block pattern was induced with warm-up phenomenon (upper panel). VT was also induced by injection of saline into the vein (middle panel). The electrogram recording from the distal tip of the CS catheter preceded QRS by $-31 \mathrm{~ms}$ (lower panel). These results strongly suggest that the focus of VT was located in the epicardium of the posterior wall in the left ventricular base.

Fig 4. Effects of adenosine triphosphate (ATP) on slow ventricular tachycardia (VT). We attempted to evaluate the efficacy of ATP on the VT using injections of mixtures of ATP with saline (upper panel) and ATP with contrast medium (lower panel). Both mixtures of ATP $(5 \mathrm{mg})$ prevented induction of VT. 


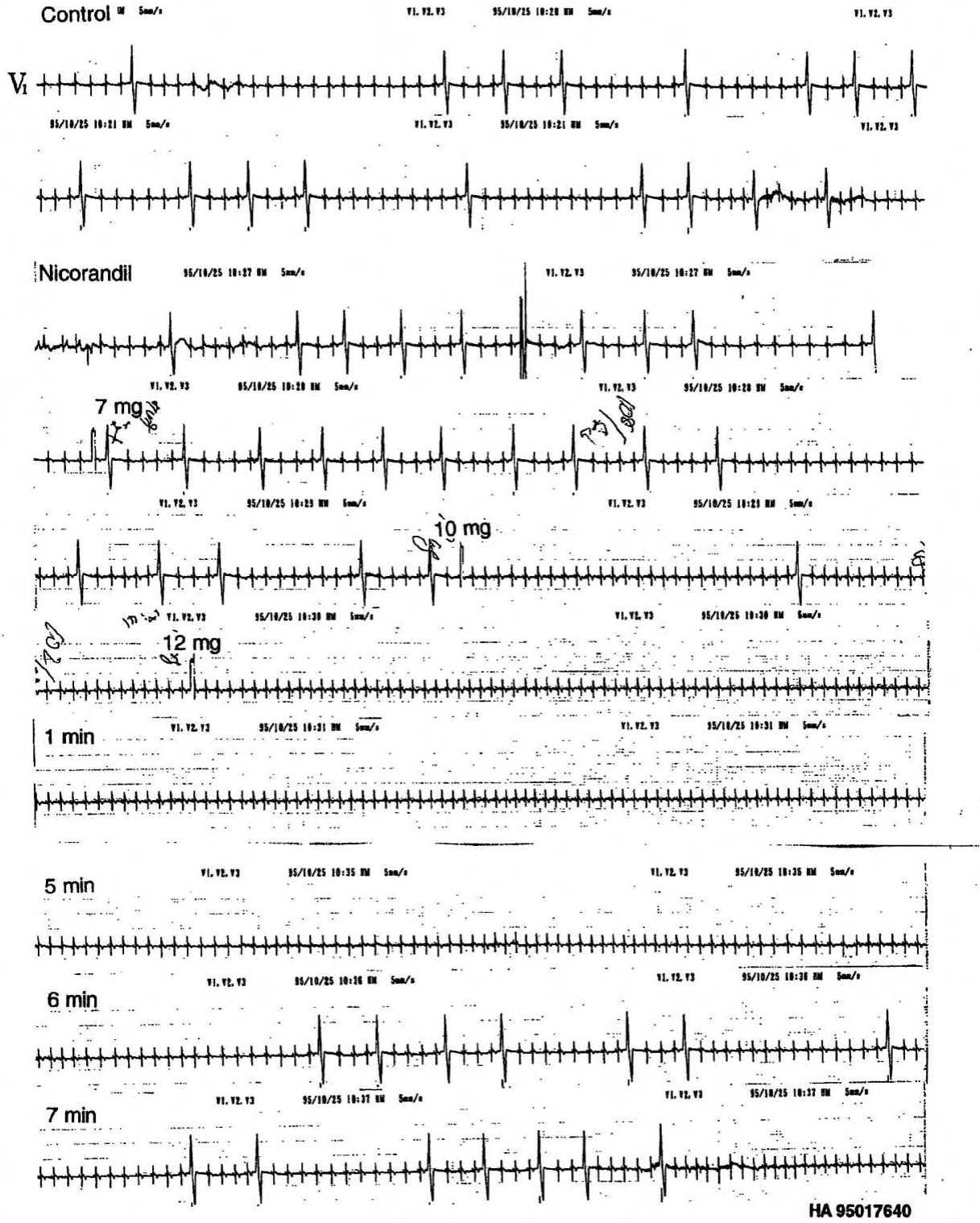

Fig 5. Effects of nicorandil on ventricular premature contractions (VPCs). As shown in lines 1 and 2, 81 VPC were observed in $10 \mathrm{~min}$ in the control state. Nicorandil $(12 \mathrm{mg})$ was infused intravenously over $3 \mathrm{~min}$. As shown in lines 5-8, after around $10 \mathrm{mg}$ of nicorandil, VPCs were being reduced and completely disappeared after $12 \mathrm{mg}$. Then, 6 min later, the VPCs reappeared in lines 9 and 10, because the half life of nicorandil is approximately 8 min. origin in the epicardium of the left ventricular base was suggested; (4) it was not induced by programmed stimulation; (5) it was not affected by isoproterenol or exercise; and (6) the VPC were prevented by nicorandil and verapamil.

\section{Mechanisms of the VT}

We suggest that the mechanism of the VT was abnormal automaticity, because the VT was not induced by programmed stimulation and induction of the VT showed the warm-up phenomenon. Induction of the VT by contrast medium may be due to a prolongation of the action potential duration (APD), which is supported by the fact that the JT intervals were prolonged after injection of the contrast medium. In an experimental study, contrast medium was reported to prolong ventricular repolarization and induce ventricular fibrillation caused by temporal dispersion of repolarization! Furthermore, in the pesent case the VT was also induced by saline. At the time, the JT intervals were also prolonged by saline injection. We consider that the epicardial hypothermia caused by injections of saline induced the VT. Antzelevitch et al reported that hypothermia prolonged the APD in the myocardium and the effects were more enhanced in epicardium than in endocardium? They suggest that the reason for the difference is a dramatic accentuation of the spike and dome morphological characteristics of the epicardial response through a decrease in the temperature from $37^{\circ} \mathrm{C}$ to $28^{\circ} \mathrm{C}$ compared with endocardium, which led to greater prolongation of the APD in the epicardium. Thus the best explanation for the induction of the VT is an increase in the $\mathrm{Ca}^{++}$current into cells through an APD prolongation, and this may induce abnormal automaticity. These results suggest the susceptibility of epicardium to induction of VT due to abnormal automaticity.

\section{Effects of ATP, Nicorandil and Verapamil on Ventricular Arrhythmia}

Previous studies have shown that adenosine- or ATPsensitive VTs do not result from re-entry, but they are 
presumed to be due to delayed afterdepolarization ${ }^{3-6}$ Adenosine antagonizes the effects of beta-adrenergic agonists in ventricular cells because it inhibits the activation of adenylate cyclase by binding to $\mathrm{P} 1$ purinergic receptors. This may inhibit the $\mathrm{Ca}^{++}$current and abolish not only triggered activity but also abnormal automaticity. Recent reports suggest that adenosine may increase IK, АTP, 7,8 which may in turn result in a decrease in the $\mathrm{Ca}^{++}$current due to a decrease of the APD, and so suppress abnormal automaticity. It supports the hypothesis that prolongation of the JT intervals after saline and contrast medium was prevented by ATP. Exogenous ATP metabolizes to adenosine immediately, and so ATP works in the same manner as adenosine. Furthermore, in the present case, VPC that had the same morphology as the VT were also inhibited by nicorandil and verapamil. To our knowledge this is the first clinical report of the effect of nicorandil on ventricular arrhythmias due to abnormal automaticity. An experimental study has reported that pinacidil, a $\mathrm{K}^{+}$channel opener, reduces the APD, and suppresses delayed afterdepolarization caused by ouabain. Abnormal automaticity induced by $\mathrm{BaCl}_{2}$ is also suppressed? Therefore we consider that nicorandil was effective on the VT in a similar manner! ${ }^{10}$ Furthermore, the efficacy of the drugs may be stronger in the epicardium than in the endocardium. Because acetylcholine produces major changes in the epicardial action potential at concentrations that have little, or no effect in the endocardium, adenosine may produce the same effects in the epicardium as acetylcholine. We conclude that some ATP-sensitive VT may come from the epicardium.

In summary, the mechanism of slow VT was suggested to be abnormal automaticity, which was due to an increase in the $\mathrm{Ca}^{++}$current into cells after prolongation of the APD induced by hypothermia, and inhibited by ATP, nicorandil and verapamil.

\section{References}

1. Murdock D, Euler D, Becker D, Murdok J, Scanlon R, Gunnar R: Ventricular fibrillation during coronary angiography: An analysis of mechanism. Am Heart J 1985; 109: 265-273

2. Antzelevitch C, Litovsky SH, Lukas A: Epicardium versus endocardium: Electrophysiology and pharmacology. In: Zipes D, Jalife J, editors. Cardiac electrophysiology from cell to bedside. Philadelphia: WB Saunders, 1990: 86-395

3. Lerman BB, Belardinelli L, West A, Berne RM, DiMarco JP: Adenosine-sensitive ventricular tachycardia: evidence suggesting cyclic AMP-mediated triggered activity. Circulation 1986; 74: $270-$ 280

4. Kobayashi Y, Kikushima S, Tanno K, Kurano T, Yazawa T, Baba T, et al: Adenosine triphosphate-sensitive ventricular tachycardia in man. Eur JCPE 1994; 4: 11-19

5. Kobayashi Y, Kikushima S, Tanno K, Kurano K, Baba T, Katagiri T: Sustained left ventricular tachycardia terminated by dipyridamole: Cyclic AMP-mediated triggered activity as a possible mechanism. PACE 1994; 17: 377-385

6. Kobayashi Y, Miyata A, Chiyoda K, Nakagawa H, Jinbo Y, Tanno $\mathrm{K}$, et al: Dipyridamole suppresses ventricular arrhythmias exhibiting catecholamine and $\mathrm{Ca}^{++}$influx sensitivity. Jpn Circ J 1996; 60: 629640

7. Kirsch G, Condinal J, Brown A: Coupling of ATP-sensitive $\mathrm{K}^{+}$channels to A1 receptors by $\mathrm{G}$ proteins in rat ventricular myocytes. Am J Physiol 1990; 259: H820-H826

8. Ito H, Tung R, Sugimoto T, Kobayashi I, Takahashi K, Katada T, et al: On the mechanism of $\mathrm{G}$ protein beta-gamma subunit activation of the muscarinic $\mathrm{K}^{+}$channel in guinea pig atrial cell membrane. J Gen Physiol 1992; 99: 961-983

9. Spinelli W, Sorota S, Siegel M, Hoffman B: Antiarrhythmic actions of the ATP-regulated $\mathrm{K}^{+}$current activated by pinacidil. Circ Res 1991; 68: $1127-1137$

10. Kobayashi Y, Shinagawa J, Asano T, Obara C, Jinbo Y, Miyata A, et al: Characteristics of idiopathic ventricular arrhythmias suppressed by ATP-sensitive K channel opener. Circulation 1997; 96(Suppl): I-152 